\title{
NASA Advanced Explorations Systems: Advancements in Life Support Systems
}

\author{
Sarah A. Shull \\ NASA, Johnson Space Center, Houston, TX \\ Walter F. Schneider \\ NASA, Marshall Space Flight Center, Huntsville, AL
}

The NASA Advanced Exploration Systems (AES) Life Support Systems (LSS) project strives to develop reliable, energy-efficient, and low-mass spacecraft systems to provide environmental control and life support systems (ECLSS) critical to enabling long duration human missions beyond low Earth orbit (LEO). Highly reliable, closed-loop life support systems are among the capabilities required for the longer duration human space exploration missions assessed by NASA's Habitability Architecture Team (HAT).

The LSS project is focused on four areas: architecture and systems engineering for life support systems, environmental monitoring, air revitalization, and wastewater processing and water management. Starting with the international space station (ISS) LSS systems as a point of departure (where applicable), the mission of the LSS project is three-fold:

1. Address discrete LSS technology gaps

2. Improve the reliability of LSS systems

3. Advance LSS systems towards integrated testing on the ISS

This paper summarized the work being done in the four areas listed above to meet these objectives. Details will be given on the following focus areas:

\section{Systems Engineering and Architecture}

With so many complex systems comprising life support in space, it is important to understand the overall system requirements to define life support system architectures for different space mission classes, ensure that all the components integrate well together and verify that testing is as representative of destination environments as possible.

\section{Environmental Monitoring}

In an enclosed spacecraft that is constantly operating complex machinery for its own basic functionality as well as science experiments and technology demonstrations, it's possible for the environment to become compromised. While current environmental monitors aboard the ISS will alert crew members and mission control if there is an emergency, long-duration environmental monitoring cannot be done in-orbit as current methodologies rely largely on sending environmental samples back to Earth. The LSS project is developing onboard analysis capabilities that will replace the need to return air and water samples from space for ground analysis. 


\section{Air Revitalization}

The air revitalization task is comprised of work in carbon dioxide removal, oxygen generation and recovery and trace contamination and particulate control. The $\mathrm{CO}_{2}$ Removal and associated air drying development efforts under the LSS project are focused both on improving the current SOA technology on the ISS and assessing and examining the viability of other sorbents and technologies available in academia and industry. The Oxygen Generation and Recovery technology development area encompasses several sub-tasks in an effort to supply $\mathrm{O}_{2}$ to the crew at the required conditions, to recover $\mathrm{O}_{2}$ from metabolic $\mathrm{CO}_{2}$, and to recycle recovered $\mathrm{O}_{2}$ back to the cabin environment. Current state-of-the-art oxygen generation systems aboard space station are capable of generating or recovering $\sim 40 \%$ of required oxygen; for exploration missions this percentage needs to be greatly increased. A spacecraft cabin trace contaminant and particulate control system serves to keep the environment below the spacecraft maximum allowable concentration (SMAC) for chemicals and particulates. Both passive (filters) and active (scrubbers) methods contribute to the overall TC \& PC design. Work in the area of trace contamination and particulate control under the LSS project is focused on making improvements to the SOA TC \& PC systems on ISS to improve performance and reduce consumables.

\section{Wastewater Processing and Water Management}

A major goal of the LSS project is the development of water recovery systems to support long duration human exploration beyond LEO. Current space station wastewater processing and water management systems distill urine and wastewater to recover water from urine and humidity condensate in the spacecraft at a $\sim 74 \%$ recovery rate. For longer, farther missions into deep space, that recovery rate must be greatly increased so that astronauts can journey for months without resupply cargo ships from Earth. 